\title{
Effect of the Cladding Layer Cavity on the Efficiency of 650 nm Resonant Cavity Light Emitting Diodes
}

\author{
Jianjun Li ${ }^{\#}$, Tao Liu, Jiachun Li, Xuan Ya \\ Key Laboratory of Opto-electronics Technology, Beijing University of Technology, Beijing, China \\ Email: "lijianjun@bjut.edu.cn
}

Received 2013

\begin{abstract}
High efficiency $650 \mathrm{~nm}$ resonant cavity light emitting diodes (RCLEDs) with a cladding layer cavity are reported. The epitaxial structure is grown with a metal-organic chemical vapor deposition (MOCVD) system. $\mathrm{Al} 0.5 \mathrm{Ga} 0.5 \mathrm{As} / \mathrm{Al} \mathrm{As}$ is used for the distributed Bragg reflectors (DBRs), and GaInP/AlGaInP multiple-quantum wells for the active region. Two RCLED samples have been fabricated, one with a cladding layer cavity and the other without. Experimental results show that the cladding layer cavity can improve the internal quantum efficiency effectively, so that an external quantum efficiency of $7.4 \%$ at $20 \mathrm{~mA}$ is reached. Meanwhile, the sample with cladding layer cavity also shows a spectral stability as the injected current changing from $20 \mathrm{~mA}$ to $100 \mathrm{~mA}$.
\end{abstract}

Keywords: $650 \mathrm{~nm}$; RCLED; MOCVD; DBR

\section{Introduction}

Because of the substrate absorption and the phenomenon of total internal reflection, AlGaInP LEDs grown on GaAs substrate suffer from a lower extraction efficiency of $2 \%$ [1]. The resonant cavity light-emitting diodes (RCLEDs) provide an effective way to improve the external efficiency by enhancing spontaneous emission and directing much light normal to the semiconductor surface [2]. Meanwhile, RCLEDs also offer many advantages over conventional LED's, such as the high temperature stability [3-5], the improved spectral purity [6] and the narrow far field beam $[7,8]$. However, experimental results of the external efficiency for RCLEDs around 650 $\mathrm{nm}$ wavelength are still not satisfied [9-12]. By now, most efforts such as special mirrors [13], photonic crystal [14] and 2-D grating [15] are made to improve RCLEDs quantum extraction efficiency, while less attention is paid to the internal quantum efficiency which is very important for RCLEDs with a narrow active region.

In this paper, we have designed and fabricated two RCLED samples with different cavity structure at 650 $\mathrm{nm}$ wavelength. For the sample with a cladding layer cavity, the internal quantum efficiency nearly reaches $100 \%$, so that an external efficiency nearly reaching the theory limits is obtained.

\footnotetext{
*Supported by the Beijing Education Committee Science and Technology Plan Surface Projects under Grant No. KM200810005002.

${ }^{\#}$ Corresponding author.
}

\section{Result and Discussion}

Figure 1 shows the RCLED structure diagram. The basic structure consists of a 34-pair Al0.5Ga0.5As/AlAs bottom N-DBR, a $1 \lambda$-cavity containing 3 quantum wells, and a 6-pair Al0.5Ga0.5As/AlAs top P-DBR. In order to form ohmic contact, there is a 5 -nm-thick $\mathrm{p}^{+}-\mathrm{GaAs}$ on the top of P-DBR. Two samples with different cavity structure are designed. For sample A, the cavity was formed by three $5 \mathrm{~nm}-\mathrm{Ga} 0.5 \mathrm{In} 0.5 \mathrm{P} / 5 \mathrm{~nm}-(\mathrm{Al} 0.5 \mathrm{Ga} 0.5) 0.5 \mathrm{In} 0.5 \mathrm{P}$ QWs centered between (A10.5Ga0.5)0.5In0.5P barrier layers, the total optical thickness of the cavity is $1 \lambda$. For sample $\mathrm{B}$, the cavity is the same as sample A except that the $1 / 4 \lambda$-(A10.5Ga0.5)0.5In0.5P-thick barrier layers near both top and bottom DBR are replaced by the $1 / 4 \lambda$ (A10.7Ga0.3)0.5In0.5P-thick cladding layers.

Three considerations have been made during the cavity design of sample B. Firstly, the thickness of each cladding layer is $1 / 4$ optical wavelength, so that the standing wave node within the cavity coincides with the (Al0.5Ga0.5)0.5 In 0.5P/ (Al0.7Ga0.3)0.5In0.5P interface to lower the optical loss. Secondly, because of the nar-row active region and the limited conduction band offset between GaInP and ( $\mathrm{A} 10.5 \mathrm{Ga} 0.5) 0.5 \mathrm{In} 0.5 \mathrm{P}$ materials, the carrier concentration within (Al0.5Ga0.5) 0.5 In0.5P bar-rier layers is not negligible. The introducing of $(\mathrm{Al} 0.7 \mathrm{Ga} 0.3) 0.5 \operatorname{In} 0.5 \mathrm{P}$ cladding layer will raise the car-rier concentration within (A10.5 Ga0.5) $0.5 \operatorname{In} 0.5 \mathrm{P}$ and QW layers by confining carriers in a narrower region, which is benefit for increasing the radiative recombinetion rate. Thirdly, the exchange of As and $\mathrm{P}$ at the Al- 
GaInP/AlAs interface will introduce many interface states which serve as the non radiative recombination centers. By using the cladding layer cavity to confine the carriers, the minority carrier concentration at the Al$\mathrm{GaInP} / \mathrm{AlAs}$ interface is lowered effectively, so that the non radiative recombination which needs two types of carriers is decreased. Shown in Figure 2 is the refractive index and the calculated longitudinal optical field intensity by the transfer matrix method [16] within sample B, also shown in the inset is the enlarged profile near the active region. The coupling between the active QW region and the cav-ity mode, and the optical field node at the $(\mathrm{A} 10.5 \mathrm{Ga} 0.5) 0.5 \mathrm{In} 0.5 \mathrm{P} /(\mathrm{A} 10.7 \mathrm{Ga} 0.3) 0.5 \mathrm{In} 0.5 \mathrm{P}$ interface can be seen clearly.

The entire RCLED structure is grown by an EMCORE D125 MOCVD system on 150 off (100) oriented n+GaAs substrate. The metal-alkyl sources used are trimethylindium (TMIn), trimethylgallium (TMGa) and trimethylaluminium (TMAl), pure $\mathrm{AsH} 3$ and $\mathrm{PH} 3$ are used as the hydride precursor gases, $\mathrm{SiH} 4$ and $\mathrm{CCl} 4$ are used as the n- and p-type doping sources, respectively, the carrier gas is $\mathrm{H} 2$ purified by $\mathrm{Pd}$ cell. V/III ratio for AlGaInP and AlGaAs is 230 and 150, respectively. The samples are grown at a temperature of $700 \mathrm{oC}$, and the wafer carrier is rotated at a speed of $1000 \mathrm{rpm}$ through-out the process.

After growth, a Ti-Au layer is deposited on the wafer top, then standard photolithograph and etching are employed to form the net pelectrode, the diameter of the bonding contact pad is $100 \mu \mathrm{m}$. The n-electrode is formed at a temperature of $400^{\circ} \mathrm{C}$ by the evaporation of AuGeNi- Au onto the backside of the substrate, which is thinned to $100 \mu \mathrm{m}$. Finally, the wafer is diced into $300 \times$ $300 \mu \mathrm{m}^{2}$ chips without encapsulating to evaluate the photoelectric performance.

Figure 3 shows the measured output light power and forward voltage versus DC current for both samples. The highly doped DBRs and the high-quality net shape top ohmic contacts result in a low voltage of $1.87 \mathrm{~V}$ at 20 $\mathrm{mA}$ for sample $\mathrm{A}$, and $1.91 \mathrm{~V}$ for sample B. An output power of $2.84 \mathrm{~mW}$ is achieved for sample $\mathrm{B}$ at $20 \mathrm{~mA}$, corresponding to an external quantum efficiency of $7.4 \%$, while the output power is only $0.85 \mathrm{~mW}$ for sample A at the same current.

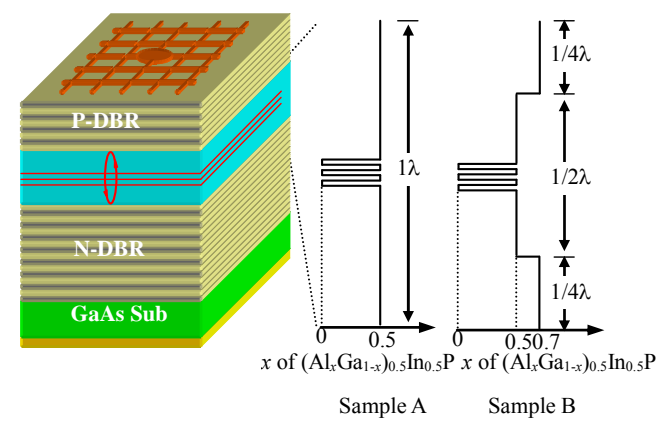

Figure 1. RCLED structure diagram.

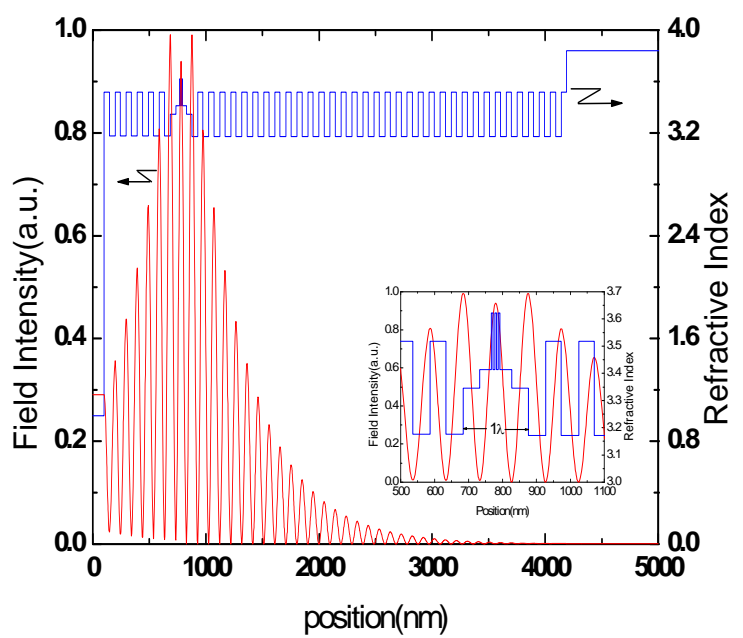

Figure 2. Refractive index and calculated longitudinal optical field intensity profile for RCLED with a cladding layer cavity.

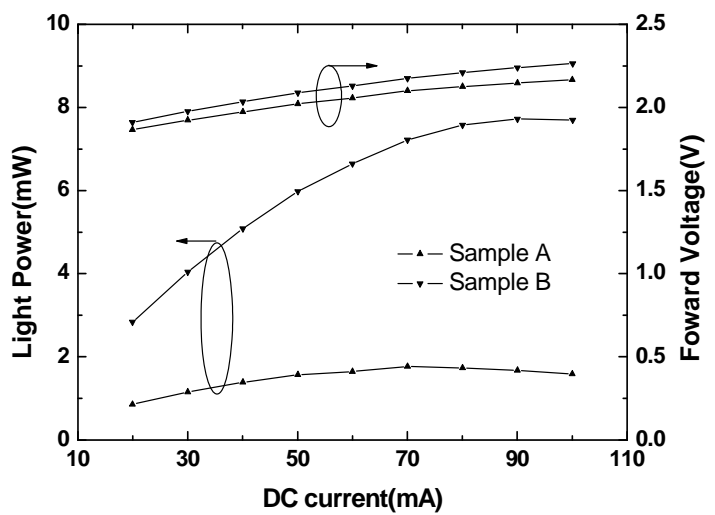

Figure 3. Light power and forward voltage versus dc current for sample $A$ and sample $B$.

The LED's external quantum efficiency $\eta_{e x t}$ is given by,

$$
\eta_{\text {ext }}=\eta_{\text {int }} \eta_{\text {extra }}
$$

where $\eta_{\text {int }}$ is the internal quantum efficiency and $\eta_{\text {extra }}$ is the extraction efficiency. For RCLEDs with DBRs as top and bottom mirrors, the extraction efficiency can be expressed as [17],

$$
\eta_{\text {extra }}=\frac{1}{m_{c}}
$$

and

$$
m_{c} \approx m_{0}+\frac{1}{2} \frac{n_{h}+n_{1}}{n_{h}-n_{1}}
$$

where $m_{c}$ is the effective cavity order including penetration length into DBRs, $m_{0}$ is the bare cavity order, $n_{h}$ and $n_{l}$ is the high and low refractive index in DBRs, respectively. According to the optical design, both sample A and sample B should have the same extraction efficiency 
of $8.5 \%$ by substituting $m_{0}=2$ and refractive index value from [18] into Equation. (2) and (3). So the large difference of the external quantum efficiency between sample $A$ and sample $B$ is due to the different internal quantum efficiency $\eta_{\text {int }}$. That is to say, the cavity structure of sample B with cladding layer benefits a higher $\eta_{\text {int }}$ by confining carriers effectively and reducing the AlGaInP/ AlAs interface recombination simultaneo-ussly. If we have considered the opaque top electrode, which amounts to $10 \%$ of the total top emitting surface, a $\eta_{\text {int }}$ of nearly $100 \%$ can be obtained for sample B.

For both samples, the optical power increases first with increasing current before it rolls-over and decreasing again due to the thermal effect. For sample B, the light power reaches its maximum point, $7.72 \mathrm{~mW}$ at a current of $90 \mathrm{~mA}$. As author's knowledge, that is the highest output power for $650 \mathrm{~nm}$ RCLEDs with the same structure. But for sample A, the maximum light power is only $1.77 \mathrm{~mW}$, meanwhile, because of the sample A's lower photoelectric efficiency and severer thermal effect, it reaches its maximum power at a lower current of 70 $\mathrm{mA}$. So we can say, because of the lower conduction band offset for AlGaInP materials and the thinner active region defined by the cavity thickness, a deliberate design of the cavity is very important for $650 \mathrm{~nm}$ RCLEDs.

Shown in Figure 4 is the electroluminescence (EL)
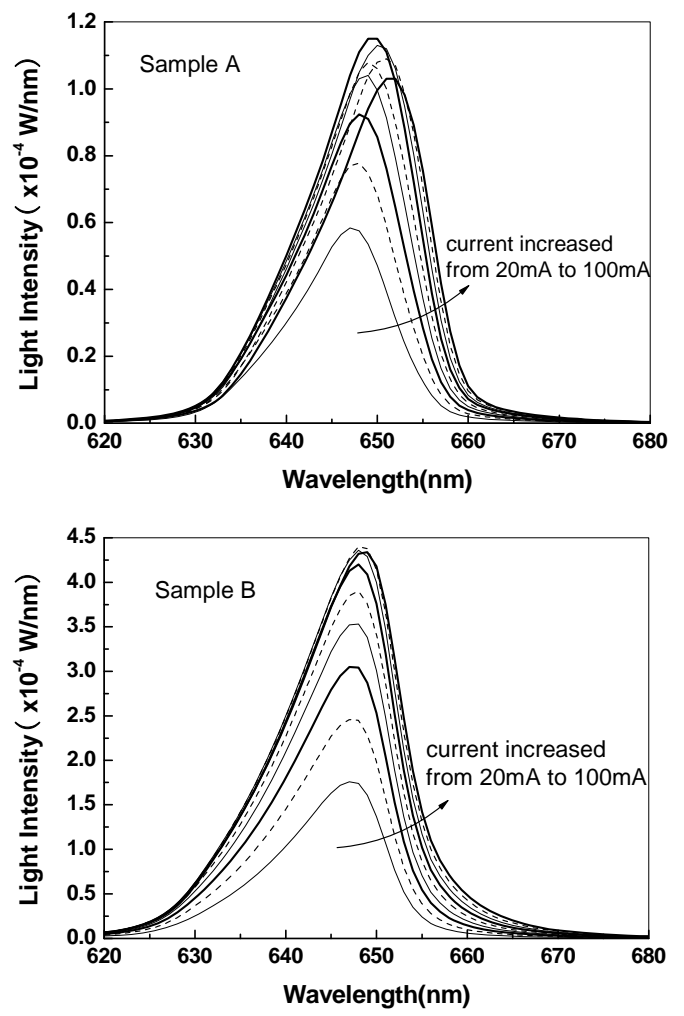

Figure 4. Emission spectrum of sample A (a) and sample B (b) at varying current between $20 \mathrm{~mA}$ and $100 \mathrm{~mA}$ with 10 mA increments. spectrum of both samples at room temperature with DC current varying between $20 \mathrm{~mA}$ and $100 \mathrm{~mA}$ (with 10 $\mathrm{mA}$ increments). Because of the high internal quantum efficiency of sample B, the peak wavelength of sample B is more stable than that of sample A. As DC current increases from $20 \mathrm{~mA}$ to $100 \mathrm{~mA}$, the peak wavelength of sample A changes $4.4 \mathrm{~nm}$ from $647.2 \mathrm{~nm}$ to $651.6 \mathrm{~nm}$, while that of sample B is only $1.2 \mathrm{~nm}$ from $647.4 \mathrm{~nm}$ to $648.6 \mathrm{~nm}$. Both samples show a same variability of the spectral purity with the current increment, that is the typical characteristics of RCLEDs [2]. When the injected current increases from $20 \mathrm{~mA}$ to $100 \mathrm{~mA}$, the full width at half maximum (FWHM) of the spectrum increases from $13 \mathrm{~nm}$ to $14.5 \mathrm{~nm}$ for sample $\mathrm{B}$, and for sample A that is from $12.5 \mathrm{~nm}$ to $13.8 \mathrm{~nm}$.

\section{Summary}

In summary, both $650 \mathrm{~nm}$ RCLED samples with different cavity structures have been fabricated. Experimental results show that for the sample with cladding layer cavity, an external quantum efficiency as high as $7.4 \%$ has been reached at $20 \mathrm{~mA}$ due to the high internal quantum efficiency, meanwhile, the sample also has a high stability of the peak wavelength and the spectral purity with the current increment. The cavity design, especially for $650 \mathrm{~nm}$ RCLEDs with AlGaInP and AlGaAs materials, is very important to get high external quantum efficiency.

\section{REFERENCES}

[1] C. P. Kuo, R. M. Fletcher, T. D. Osentowski, M. C. Lardizabal and M. G. Craford, "High Performance AlGalnP Visible Light-Emitting Diodes," Applied Physics Letters. Vol. 57, No. 27, 1990, 2937. doi:10.1063/1.103736

[2] E. F. Schubert, Y. H. Wang, A. Y. Cho, L. W. Tu and G. J. Zydzik, "Resonant Cavity Light-Emitting Diode," Applied Physics Letters, 1992, Vol. 60, No. 8, 106489. doi:10.1063/1.106489

[3] Y. A. Chang, C. L. Yu, I. T. Wu, H. C. Kuo, T. C. Lu, F. I. Lai, L. W. Laih, L. H. Laih and S. C. Wang, "Design and Fabrication of Temperature-Insensitive In GaP-InGaA IP Resonant-Cavity Light-Emitting Diodes," 2006 IEEE Photonics Technology Letters, Vol. 18, No. 16, 1690. doi:10.1109/LPT.2006.879931

[4] J. R. Chen, T. S. Ko, T. C. Lu, Y. A. Chang, H. C. Kuo, Y. K. Kuo, J. Y. Tsai, L. W. Laih and S. C. Wang, "Fabrication and Characterization of Temperature Insensitive $660 \mathrm{~nm}$ Resonant-Cavity LEDs," IEEE Journal of LightWave Technology, Vol. 26, No. 13, 2008, 1891. doi:10.1109/JLT.2008.920639

[5] H. Fardi, M. Jan, B. Van Zeghbroeck, 2012, Photovoltaic Specialists Conference. 16785

[6] E. F. Schubert, N. E. J. Hunt, R. J. Malik, M. Micovic and D. L. Miller, "Temperature and Modulation Characteris- 
tics of Resonant-Cavity Light-Emitting Diodes," IEEE Journal of Lightwave Technology, Vol. 14, No. 7, 1721. doi.org/10.1109/50.507950

[7] R. Joray, M. Ilegems, R. P. Stanley, W. Schmid, R. Butendeich, R. Wirth, A. Jaeger and K. Streubel, "Far-Fields Radiation Pattern of Red Emitting Thin-Film Resonant Cavity LEDs," IEEE Photonics Technology Letters, Vol. 18, No. 9, 2006, 1052. doi.org/10.1109/LPT.2006.873553

[8] S. Morikura and Y. Okamura. "Angle-Dependent Spectral Width of Resonant-Cavity Light-Emitting Diode," IEEE Photonics Technology Letters, Vol. 21, No. 10, 2009, 21 660. doi.org/10.1109/LPT.2009.2016218

[9] S. W. Chiou, Y. C. Lee, Y. C. Yang, C. S. Chang and T. P. Chen, "High-Performance Resonant Cavity Light-Emitting Diode for Plastic Optical Fiber Application," Proceeding of SPIE, Vol. 5366, 2004. doi.org/10.1117/12.525285

[10] M. M. Dumitrescu, M. J. Saarinen, M. D. Guina and M. V. Pessa, "High-Speed Resonant Cavity Light-Emitting Diodes at $650 \mathrm{~nm}$," IEEE Journal on Selected Topics in Quantum Electronics, Vol. 8, No. 2, 2002, pp. 219-230. doi:10.1109/2944.999174

[11] J. J. Li, Z. Yang, J. Han, J. Deng, D. S. Zou, Y. Z. Kang, L. Ding and G. D. Shen. Acta Physica Sinica. 2009, 58 6304.

[12] R. Wirth, C. Karnutsch, S. Kugler, S. Thaler and K. Streubel. "Red and Orange Resonant-Cavity LEDs," Proceedings of SPIE, Vol. 4278, 2001. doi: $10.1117 / 12.426855$

[13] P. H. Lei and C. D. Yang. Solid- State Electronics, 2008, 52227.

[14] K. Berggenek, C. Wiesmann, H. Zull, R. Wirth, P. Sundgren, N. Linder, K. Streubel, and T. F. Krauss. "Directional Light Extraction from Thin-Film Resonant Cavity Light-Emitting Diodes With a Photonic Crystal," Applied Physics Letters, Vol. 93, No. 23, 2008, 231109. doi: $10.1063 / 1.3046130$

[15] J. Rao, R. Winfield and S.O' Brien. IEEE Photonics Technology Letters. 2009, "Enhancement of Light Extraction From Resonant Cavity Light-Emitting Diodes Using a 2-D Grating Embossed in $\mathrm{TiO}_{2}$ Sol-Gel," Vol. 21, No. 13,941 . doi:10.1109/LPT.2009.2020808

[16] J. J. Li and G.D. Shen. Journal of Optoelectronics. Laser. 2006, 171457.

[17] H. Benisty, H. D. Neve and C. Weisbuch. "Imoact of Planar Microcavity Effects on Light Extraction-Part I : Basic concepts and Analytical Trends," IEEE Journal Quantum Electron, Vol. 34, No. 9,1998, pp. 1612-1631. doi.org/10.1109/3.709578

[18] S. Gehrsitz, F. K. Reinhart, C. Gourgon, N. Herres, A. Vonlanthen and H. Sigg, "The Refractive Index of AlxGa1-xAs Below the Band Gap: Accurate Determination and Empircal Modeling," Journal of Applied Physics, 2000, Vol. 87. 7825.doi:10.1063/1.373462 